\title{
Histoires de Pulotu
}

\section{Claire Moyse-Faurie}

\section{(2) OpenEdition}

\section{Journals}

Édition électronique

URL : http://journals.openedition.org/jso/603

DOI : $10.4000 /$ jso. 603

ISSN : 1760-7256

Éditeur

Société des océanistes

Édition imprimée

Date de publication : 1 décembre 2006

Pagination : 141-151

ISSN : 0300-953x

\section{Référence électronique}

Claire Moyse-Faurie, «Histoires de Pulotu», Journal de la Société des Océanistes [En ligne],

122-123 | Année 2006, mis en ligne le 01 décembre 2009, consulté le 01 mai 2019. URL : http:// journals.openedition.org/jso/603 ; DOI : 10.4000/jso.603

(c) Tous droits réservés 


\title{
Histoires de Pulotu
}

\author{
par
}

Claire MOYSE-FAURIE*

\section{RÉSUMÉ}

Pulotu, domaine des dieux ou lieu de séjour des morts, est un toponyme connu aussi bien en Polynésie qu'à Fidji. On ne sait s'il s'agit d'un lieu entièrement mythique ou de la réminiscence d'un endroit autrefois prospère. La tradition orale s'attache à relater sa richesse, les faits et gestes de ses habitants, et la façon dont on peut y séjourner de façon transitoire. Les deux textes wallisiens présentés ici ont été enregistrés en 2000 auprès d'Alikisio Liufau, du village de 'Utufua à Wallis.

Mots-CLÉs : tradition orale, mythe, Pulotu, domaine des dieux, Fidji, Wallis, Futuna.

Nombreuses sont les évocations de Pulotu dans les récits des premiers missionnaires, qu'ils aient résidé en Polynésie occidentale ou à Fidji, et les traditions orales encore vivaces à l'heure actuelle relatent diverses aventures qui lui sont liées. Pulotu y est souvent décrit comme le domaine souterrain - parfois sous-marin - des dieux, des esprits ou des morts. On y trouve toutes sortes de richesses et les vivres y abondent. Certains récits y placent l'origine du kava, d'une igname Dioscorea alata ou d'un taro Colocasia esculenta. On y pénètre par un trou sous la mer, visible à marée basse, en atteignant la ligne d'horizon, là où la mer rencontre les nuages, ou encore par une grotte située dans l'arrière-pays.

\section{ABSTRACT}

Pulotu, residence of the gods or sojourn of the dead is a placename known in Polynesia as well as in Fiji. It is not known whether it is an entirely mythical place or the remembrance of a formerly prosperous place. Oral tradition tells us about its wealth, the way of life of its inhabitants, and how you can stay there for a short while. The two Uvean texts presented here were recorded in 2000 from Alikisio Liufau who lives in 'Utufua village in Wallis.

KEYWORDS: oral tradition, legends, Pulotu, residence of the gods, Fiji, Wallis, Futuna.

À partir de ces légendes tongiennes, fidjiennes, samoanes, futuniennes ou wallisiennes, plusieurs localisations ont été proposées pour Pulotu. L'ethnologue Edwin Burrows (1936 : 103 et 1937 : 85) reprend les indications fournies par les pères Bataillon et Henquel (tous deux en poste à Wallis), selon lesquels Pulotu serait une terre située à l'ouest. En accord avec cette hypothétique origine située à l'ouest de la Polynésie occidentale, le linguiste Paul Geraghty (1993) a tenté de trouver une véracité historique à ce mythe de Pulotu et l'a localisé à Fidji. Pour Paul Geraghty, Pulotu faisait partie de Matuku, île située à $19^{\circ}$ de latitude, au sud de Taveuni dans l'archipel fidjien, et donc légèrement au sud-ouest de

* Linguiste, umR 7107 Lacito-CNRS, moyse@vjf.cnrs.fr 
Futuna. À Fidji, Pulotu correspondrait à Burotu, qui aurait été autrefois un centre important de commerce, un lieu où s'échangeaient toutes sortes de produits exotiques. L'île Burotu, aussi nommée Vanuakula, «le pays rouge », à cause du commerce des fameuses plumes rouges d'oiseaux, aurait été submergée. Vengeance des dieux suite à l'exécution d'un cochon blanc, tremblement de terre, montée des eaux..., sa disparition aurait nourri la légende. Toujours selon Paul Geraghty, Burotu aurait aussi été un grand centre culturel, ce qui expliquerait le second sens du mot pulotu en futunien, "maitre de danse " ou, d'après Bataillon, en wallisien, «celui qui entonne dans les chants des danses ».

Les héros civilisateurs, tels le Muni d'Alo à Futuna, alias Ufigaki à Sigave (Frimigacci et al., 1995), ou la déese Lupe qui, métamorphosée en colombe, permit l'instauration du premier roi d'Alo en apportant aux hommes le fameux kete 'uli, " panier noir», seraient venus du royaume des dieux.

À Tonga, le roi de Pulotu est nommé Havea Hikule'o, connu à Samoa comme Savea Si'uleo (Hovdhaugen, 1987 : 190) et, à Wallis, d'après le père Henquel, sous celui de Hikule'o. Mi-dieu mi-déesse, Havea Hikule'o, née, d'après les légendes tongiennes, de l'union incestueuse de Taufulifonua et de Hanelolofonua, aurait hérité de Pulotu, qui aurait fait partie alors de l'île Tongamama'o, terre à partir de laquelle auraient été peuplées les îles de Polynésie occidentale.

Dans les deux récits présentés ici, le roi du domaine des Dieux est simplement désigné par Tu'i Pulotu, « chef de Pulotu ». Ces récits ont été enregistrés à 'Utufua (Mu'a, Wallis) auprès de Alikisio Liufau, le 11 mars 2000, puis transcrits et traduits avec l'aide de Franck Asitalatini. Le premier raconte les exploits de Maka la Pierre, fils de Maui, originaire de 'Utufua, village du narrateur. Maka sortira vainqueur de toutes les épreuves imposées par Tu'i Pulotu, certes grâce à son intelligence et sa force, mais surtout grâce aux incroyables capacités des compagnons qu'il s'est choisis: Feke le Poulpe champion de l'apnée, Mapula l'Enflé capable d'engloutir des festins et une énorme quantité de kava, et Toke l'Anguille, le plus rapide des nageurs ${ }^{1}$. Tu'i Pulotu, vaincu, cèdera toutes ses richesses à Maka et mettra fin à l'existence de Pulotu. Comme le suggère Paul Geraghty (1993), les richesses de Burotu, convoitées par les îles voisines, furent vraisemblablement la cause de guerres de conquête qui mirent fin à son rayonnement commercial et culturel, dans l'hypothèse où cette île aurait une réelle existence géographique.

Le second récit, du même narrateur wallisien, prend place à Futuna. Il s'agit à nouveau d'une visite au Pulotu, sorte de monde sous-marin que l'on atteint en pénétrant dans un trou en bord de mer, à marée basse, à condition d'être initié par un de ses habitants. La rencontre entre le futunien Tomasi, homme de Taoa, et l'homme de Pulotu a lieu de nuit, alors que Tomasi, las de se faire voler ses récoltes, décide de rester surveiller son champ. L'homme de Pulotu admet ses larcins et entraîne le peu rancunier Tomasi à Pulotu, qui se révèle être un lieu rempli de richesses et de biens, sans doute le fruit de nombreux pillages. Bravant un interdit, Tomasi peut observer les allées et venues des gens de Pulotu, puis est reconduit sur son île, où se prépare une cérémonie de deuil. Il comprend alors que les siens l'ont cru mort et que c'est de son enterrement qu'il s'agit. Fort heureusement, il peut prouver qu'il est bien vivant et raconte ses aventures. Mais l'entrée de Pulotu a disparu à jamais. Ce second récit est connu à Futuna comme l'histoire de Malamailagi (Frimigacci et Hardy, 1996), lui aussi victime de voleurs, et qui aurait accepté de se rendre au Pulotu. Là, on lui donna le choix entre " une très belle fille assise sur une natte " ou une surprise. Malamailagi choisit la surprise qui se révéla être Falekula, " maison rouge », la propre fille du roi de Pulotu et de la déesse Lupe. Mais de retour à Taoa, Malamaitagi dédaignera Falekula pour une étrangère à cheveux clairs et Lupe quittera Futuna. À l'appui de cette origine futunienne de Pulotu, sur les plateaux entre Taoa et Leava, Daniel Frimigacci et Maurice Hardy ont relevé différents toponymes : le sommet Le Feke, « la pieuvre »; Saufekai, nom du roi cannibale de Pulotu ; Savaiki, correspondant évidemment à l'Hawaiki de la Polynésie orientale; ou encore Vai o Lupe, synonyme de Vaiola, le lac de l'éternel jeunesse décrit par le père Servant (1995:68).

Il y aurait donc peut-être autant de Pulotu/Hawaiki que de terres d'origine, chacun exprimant le même concept, véhiculant les mêmes symboles de "paradis perdu ", de lieu ancestral plus riche, plus merveilleux que les îles

1. Les récits mettant en scène des serviteurs de chefs dotés d'extraordinaires capacités sont récurrents en Océanie, particulièrement en Polynésie, mais aussi en Nouvelle-Calédonie. Ainsi, par exemple, l'histoire de Deekwââxiti, ancêtre-dieu de plusieurs clans de la région Xârâcùù (Canala), parti récupérer sa fille enlevée à Ouvéa, avec l'aide de ses serviteurs-héros qui réussissent à déjouer toutes les embûches : Rat de Forêt creuse le tunnel qui leur permet de s'enfuir en trompant la vigilance des ravisseurs, Rat Blanc ronge les amarres qui retiennent leur pirogue, Ver Luisant éclaire les nuits, Oiseau de Forêt calme le mauvais vent et Oiseau-Suceur aspire les énormes vagues qui menacent de les engloutir (Moyse-Faurie, 1995). 
plus à l'est habitées par la suite, un lieu idéalisé d'où l'on aurait été chassé par des guerres meur- trières et que l'on cherche à reconquérir à travers un processus de mythification.

\section{Rencontre entre Maka, fils de Maui, et Tu’i Pulotu}

'E tou talanoa nei ki te foha o Māuĩ.

Ko Màuī ne'e iai tona foha ko Maka.

Pea ko Māuì ne'e ko te tagata i'Utufua nei.

Pea ko tona nofo'aga ko te 'api ko Fanātakotō.

Pea ne'e nofo ai tona foha 'aia ko Maka.

Pea nofonofo ia Maka, kae ko tona vai ma'anu ko te vaitufu, 'e higoa ko Vaikalapu.

Hoko lā 'aia ki te tahi afiafi pea hifohifo leva ia Maka i tona 'api, Fanātakotō,

kae ko te afiafi 'aia 'e tō atu pē te la'à o malu te pō'uli kae hopo ake te māhina.

Pea 'e hifo tonu ifo lā ia Maka kae hopo ake te māhina.

Pea sio ia ki te 'alu ake a te māhina, 'e iai te tagata,

'e 'alu ki te māhina, kae ha'u ia i te fuga tai.

'E sio ia ki te ha'u a te tagata 'e fakavilivili pea toe fakavilivili mai mo ia ki te vai,

nā pātō fakatahi lā ki te vaitufu, nā pātō 'aia.

Ha'u te tagata 'e ina 'ilo'i te higoa o te tama 'aē ko Maka

“Mālō Maka, sìou mālōlō mo tau kātaki pē i te fenua nei”.

“'ēi, mālō tau folau mo si'au ia faiga mai, ko tatā hoki felāve'i $\bar{e}$ ?’’

Pea 'ui age leva e te tagata' aē:"ne'e ha'u he ko'e ko koe 'aē 'e ha'u ki toku vai?',

Pea tali atu ia e Maka: "ko toku vai".

"'E ke 'ui mai ko tou vai he ne'e ko tou vai mai fea?"

"'E ke ha'u mai fea? Ko toku vai."

Fai tanā fihi, fihi fihi 'aia pea nā kē foki.

Mafuli atu ia Maka o ma'u atu te lau'ulu o te tagata o takai, takai o sī ki ’oluga.

Tō ifo te tagata o tō kehe ia i te maka 'aē nā fihi ai.

Pea alamai te tagata ia kiā Maka, ma'u tona lau'ulu, takai o sī ki 'oluga.

Tō ifo Maka 'aia o tō tu'u ki te maka.

Pea alamai leva te tagata he ne'e ha'u o 'alofa:

“Mālō Maka tau kātaki. Kua hoki au 'ilo’i ko tou vai.

Pea ke ha'u lā o nofo he ko te gata 'aē o tatā felāvei.

Kae 'e au kole atu kiā koe ke ke 'eva'eva ifo ki toku fenua ko Pulotu, ke tã felāve'i ai." 'Io."

“'ēi, tā fai ki ai. 'Alu lā koe." Pea ina 'ui mai :
Je vais vous raconter l'histoire du fils de Maui.

Maui avait un fils, Maka.

Et Maui était originaire d'ici, de 'Utufua.

Sa résidence était le domaine de Fanatakoto.

Et c'est là que demeurait son fils Maka.

Maka vivait là, et il avait l'habitude d'aller se laver à la source de Vaikalapu.

Un soir, Maka descendait tranquillement de son domaine, Fanatakoto,

à l'heure où le soleil se couche pour laisser la place à la nuit et au clair de lune.

C'est d'ailleurs juste au moment où la lune commençait à se lever que Maka se mit en route.

Et c'est grâce à la lune qui se levait qu'il vit qu'il y avait un homme

sous le clair de lune qui avançait à la surface de la mer. Il vit que l'homme se déplaçait rapidement alors, il se pressa lui aussi vers l'eau

et tous deux arrivèrent ensemble à la source, ils arrivèrent là.

L'homme qui venait d'arriver connaissait le nom du garçon, de Maka :

"Bonjour, Maka, bonne santé et bon courage pour toi ici-bas!"

"Merci, bonjour à toi, voyageur, mais il me semble que nous nous rencontrons pour la première fois, non?"'

L'inconnu lui dit : "Comment se fait-il que tu viennes ici à ma source?"

Maka lui répondit : Mais c'est ma source !"

"Pourquoi dis-tu que c'est ta source, depuis quand serait-ce la tienne?"

"D'où venez-vous ? C'est ma source!'

Ils se disputèrent tant qu'ils en arrivèrent aux mains.

Maka saisit l'homme par les cheveux, le fit tournoyer, tournoyer et le projeta en l'air.

L'homme retomba hors du rocher où se déroulait le combat.

Il tendit les mains pour saisir Maka par les cheveux, le fit tournoyer pour le lancer en l'air.

Maka retomba bien comme il faut sur ses pieds, sur le rocher.

L'homme tendit alors les mains pour le saluer :

'Bravo, Maka, tu as gagné. Je sais désormais que c'est ta source.

Tu peux y rester, car notre rencontre arrive à son terme.

Toutefois, je t'invite à venir te promener dans mon pays, Pulotu, afin de nous rencontrer à nouveau." "D'accord", répondit Maka.

"Bien, faisons comme cela, viens me voir". Et l'homme ajouta : 
“Kau fakakātoa atu tou higoa Maka he kua au 'ilo’i,

kua tu'afia mai taku ha'u, mole au felāve'i mo he tagata $i$ henī.

Ko tatā hoki felāve'i è, pe au 'ilo'i leva ko koe ko te tagata.

Ko tou higoa ko Fakafu'umaka pea ko tou fu'u maka 'aia mole toe lava'i ia e he tahi."

Pea nā tau'inē.

'Alu ia kae ina 'ui mai: "'eva'eva ifo ki Pulotu, tā felāve'i ki ai.",

Pea tali atu pē ia e Maka: "'e tā fai ki ai 'alu lā koe, 'e au 'eva'eva atu."

Ko te 'alu 'aia a te tama kae 'alu ake Maka o moe pea 'alu ake ia kae mole kei galo tanā felāve'i mo te temonio.

Pea 'alu leva ia ki Faioa.

'E nofo ai tana 'ū kui ko Lagi mo Lalofenua.

Fehu'imai e tana' ù kui : "Oie Maka, ko'e ko koe ia 'aē,

ko tau hoki tuli mai ē." "'E au ha'u au kiā koulua."

"'èi, koteā 'e iai hau logo ?"

“Ko taku fekau ko au 'aenī ne'e felāve'i anapō mo te tagata ki toku vai 'aē ne'e koulua gaohi.

Felāve'i ai māua ki ai anapōi te hopo 'aē a te māhina.

Pea mā gao'i ai pea ne’e au mālō.

Pea mā tau 'alofa leva mo te tagata pea ne'e ina kole mai kiā au ke au 'eva'eva ifo ki Pulotu.

Ko ia 'aē mole au 'ilo'i foki au Pulotu pē tu'u i fea.

Pea mole au 'ilo'i he vaka ke au 'alu ai.",

“Pea 'ui age leva e tana' 'ù kui sio mai, 'alu koe o fìmālie.

Tuku he mā fai tou vaka ke ke 'alu ai.

Hake koe ki 'uta."

Ha'u ai te tama 'aia ki tona 'api

kae omai leva tana 'ū kui i te ava 'aē ko Avahugalu o to'o mai ai maka e lua.

Omai 'aia o lagolago leva i te 'utu'aenī i Fagalele ; ko te vaka'aia

Ko te vaka ia kā' 'alu, ko te 'ū maka...

'Osi lagolago pea fekau ai e tana 'ū kui kiā Maka ke olo $l \bar{a}$

o laga ifo te ifi ne'e tu'u i Katanoa, ko te ifi māhaga ko te ifi kula mo te ifi 'ui.

Ko te tafito e tahi kae kula tona fua pea 'ui.

Ko te là 'aia o te vaka.

Pea 'ui age leva e tana 'ū kui : "Maka, ko'enī tou vaka. Pea fili lā te kau folau ke 'alu mo koe."

Fili leva te kau folau ko te tagata ko Mapula peamo te tagata ko Toke, peamo te tagata ko Feke
"Je tiens à compléter ton nom, Maka, car je reconnais que

depuis le temps que je viens ici, je n'y avais encore rencontré aucun homme.

C'est notre première rencontre, et je sais à présent que tu es vraiment un homme.

Tu t'appeleras désormais Fakafu'umaka, et ta pierre, personne ne pourra la déplacer."

Ils se dirent au revoir.

En partant, l'homme dit encore : "Viens te promener à Pulotu, ainsi nous nous reverrons."

Maka lui répondit : "Entendu, va, j'irai faire un tour chez toi."

Après son départ, Maka alla se reposer

mais il n'oublia pas sa rencontre avec le démon.

Il se rendit ensuite à Faioa.

C'est là qu'habitaient ses grands-parents Lagi et Lalofenua, Ciel et Terre.

Ses grands-parents lui demandèrent : "Tiens, Maka, quel bon vent t'amène?

C'est maintenant que tu arrives !" "Je suis venu vous voir tous les deux."

"Qu'y a-t-il, qu'as-tu à nous annoncer ?',

“Ce que j'ai à vous dire, c'est qu'hier soir, j'ai rencontré un homme à la source que vous avez aménagée.

Nous nous sommes rencontrés hier soir au moment où la lune se levait.

Nous nous sommes battus et j'ai gagné.

Nous nous sommes ensuite dits au revoir et il m'a demandé de venir lui rendre visite à Pulotu.

Cependant, je ne sais pas vraiment où se trouve Pulotu.

Et je n'ai pas non plus de pirogue pour y aller.'

Ses grands-parents lui dirent: "Écoute, va te détendre.

Ne t'inquiète pas, nous allons te fabriquer une pirogue pour que tu puisses partir.

Retourne là-haut chez toi."

Le garçon retourna donc chez lui

tandis que ses grands-parents se rendaient à la passe de Avahugalu pour y ramasser deux pierres.

Ils allèrent les poser sur le rocher de Fagalele ; c'était en fait la pirogue.

C'était le bateau qui devait naviguer, ces pierres.

Une fois les pierres déposées, les grands-parents dirent à Maka qu'ils allaient

arracher un châtaignier ${ }^{2}$ de Katanoa, un châtaignier jumeau, mi-rouge, mi-vert.

Les pieds jumeaux étaient de la même souche, mais l'un portait des fruits rouges et l'autre des fruits verts. Ils devinrent la voile de la pirogue.

Ses grands-parents dirent à Maka : "Voici ta pirogue. Tu dois choisir à présent ton équipage."

Furent choisis pour l'équipage : Mapula « l'Enflé », Toke « l'Anguille », Feke « le Poulpe », 
peamo te tagata ko Fakafu'umaka peamo ia Maka

'Aēe fakatu'u te ifi ki te fuga vaka pea 'ui mai e tana 'ū kui :

“Kā agiagi atu pēe 'e hivahiva atu pēe māua te ki'i matagi o tokoutou vaka

pea koutou pikipiki ma'u kae 'alu te vaka.'

'Ui mai e tana 'ū kui agi te matagi mai Kulufau pea folau te vaka.

Ko te 'alu atu 'aenā a te vaka o tu'u 'osi i te potu 'aenā i Mala'e, 'aē i Nukuatea.

Tu'u'osi atu ai kae toe tō atu ki tai o toe fagona te vaka. 'Alu'alu'aia te vaka, pea 'ui age leva e Maka :

"ko tatatou 'alu 'aenī'e tou 'alu ki fea?"

Pea 'ui mai ia e Toke: "'a'ahi, tuku koe te vaka ke 'alu! Pea kā au 'ui atu pē ke tokaga,

pea ke tokaga koe he ko koe 'aè 'e pailate.

'E tou olo atu 'aen̄̄ 'e iai pē te me'a 'e ma'anu pē i te fuga tai.

Pea he'eki nei lave'i ia tātou.

Pea kā tou ovi pē 'aia, pe au 'ui atu ke fakalaga te vaka i tona fo'i tu'a.

'Alu atu foki lā pea 'ui mai e Toke ko'en̄̄ kua ovi.

Ha'u koe ke laga te vaka i te fo'i tu'a.

Ko te 'alu atu pēe 'aia te vaka laga i te fo'i tu'a 'aēo te me'a foki 'aē 'e ma'anu. Mou te me'a.

Mou ifo 'aia, 'alu atu, hifo tonatou vaka ki Pulotu.

Olo atu là nātou, kua tokalelei he kua 'ilo'i te 'alu ifo a te vaka.

Kua fakatahi te fenua 'aia mo teuteu ia mo te kai kātoaga.

Pea tō atu pē 'aia te folau o fai te tali folau o fakaha'uha'u,

pea 'ui atu e Maka, pea kua tala mai mo te higoa o'ona ko ia Tu'i Pulotu

Pea 'ui atu leva e Maka : 'Mālō pē, Tu’i Pulotu, siau fakatalitali mo tau 'ü haha'i.

Ko'enī foki ne'e mole au fia si'aki tau kole,

ne'e ke kole mai ke tà felāve'i mai ki henī.

Ko ia 'aenī 'e au ha'u ai, na'a ke 'ui e au tuku tālaga.",

“'ēi, 'e lelei 'osi ia Maka tau ha'u ke ke sio koe ki to'oku fenua mo ta'aku haha' $i$.

'èi, kua tonu ke tā felāve' ',', pea 'uluaki lea mai leva Tu'i Pulotu:

“Maka, 'e hoki fakamuli he kava, kae tauhi ma'uli mu'a!

Ko te kātoaga 'aenā 'e tuku mai tu'a, ko te kātoaga kiā koe mo tau kau folau.

Pea ko tona lea pēe 'e 'avatu, kai ke 'osi.

Na'a mole 'osi kà koutou pupuli foki i henī.'

Pea tali pē ia e Maka: "'e fai pē ki ai Tu'i Pulotu.

Tuku pe ke faifaitaki he me'a 'e feala."

Pea hekeheke mai leva Mapula: "Maka,

vaka'i pè koe he tokotahi ke 'alu o 'aumai hakoutou ki' i 'umu ki fale nei.

He ki'i me'a pée 'e koutou lava'i et bien sûr Fakafu'umaka « Pierre énorme », alias Maka.

Ils dressèrent le châtaignier au-dessus de la pirogue et les grands-parents leur dirent :

"Lorsque nous nous mettrons à souffler, le vent gonflera la voile de votre pirogue,

il faudra bien vous accrochez car le bateau va filer."

Les grands-parents dirent alors : "Que le vent souffle de Kulufau, que vogue la pirogue".

La pirogue fila jusqu'à Mala'e, sur Nukuatea.

La pirogue atteignit Nukuatea puis reprit sa route.

La pirogue poursuivait sa course, et Maka demanda :

"Mais où allons-nous donc?"

Toke lui répondit : "Mais laisse donc faire la pirogue ! Cependant, lorsque je te dirai de faire attention,

alors tu feras bien attention car c'est toi le capitaine.

Sur notre chemin, il y aura une bête nageant à la surface.

Elle ne connait pas encore notre existence.

Lorsque nous serons à proximité d'elle, je te dirai de faire passer la pirogue sur son dos."

Au moment prévu, Toke dit : "Nous y voilà !

Dirige-toi sur la bête et pose la pirogue sur son dos !"

La pirogue se posa exactement sur le dos de la bête qui nageait. Cette dernière se noya.

La bête se noya, et leur pirogue put descendre vers Pulotu.

Lorsqu'ils arrivèrent, tout était déjà prêt car on était au courant de leur venue.

Le peuple de Pulotu tout entier s'apprêtait à les recevoir avec un grand festin.

Les voyageurs firent les salutations d'usage,

Maka prit la parole, apprit que leur hôte s'appelait Tu'i Pulotu

alors il lui dit : "Merci pour ton accueil et celui de ton peuple.

Je n'ai pas eu envie de refuser l'invitation

que tu m'avais faite à venir te retrouver ici.

C'est pour cela que je suis venu, afin que tu ne dises pas que je ne suis pas de parole."

"Oui, tu as eu raison, Maka, de venir voir mon pays et mon peuple.

Oui, il est bon de nous rencontrer." Puis Tu'i Pulotu donna les premières explications :

"Maka, nous terminerons par un kava, mais passons d'abord à table !

Le festin disposé là dehors, c'est pour toi et tes compagnons.

Tout ce que je vous demande, c'est de tout manger.

Si vous ne finissez pas tout, alors vous serez perdu."

Maka lui répondit : "Entendu, Tu’i Pulotu,

Nous allons faire notre possible."

Mapula s'approcha de Maka et lui dit : "Maka, choisis quelqu'un pour aller chercher un peu à manger pour vous dans la maison.

Vous viendrez à bout de ces quelques vivres 
kae tuku kau hōhō atu ki tu'a o vaka'i te potu kātoaga na'a 'e tuku mai ai."

"Fai ia ki ai !"

Ko ia 'aia pea ha'u ia Toke o hiki age pē tanatou ki'i puaka e tahi

kae hū atu leva Mapula o nofo mai i muli o te kātoaga.

Te haga 'aia a Mapula o hiko mai te kātoaga, te puaka,

te magisi, te 'akau'aē ne'e leta ai mo te tau mohomoho.

Fagona fuli ki loto.

Pea'osi pea tōatu leva ki te tai o fakamānava i tana kai. 'Oi kua moaloā foki te fenua.

Mole kei 'ilo'i e Tu'i Pulotu he pule'aki foki ia.

'Osi fakamānava 'aia e Mapula tana kai, pea 'alu ake ki te fakatahi.

Pea fai leva te fakamālō 'aè a Tu'i Pulotu :

" Maka mālō tau kātaki kua ke ma'uli.

Kei toe te tahi faiva : ko ia 'aen̄̄' e fakahā atu kiā koe, ko tātou ko te tuli moana,

ko te fai kāuga 'aē pe ko ai 'aē kāuga vave.

Pea ko ta'aku tokotolu pea ke filifili pē koe pe ko tau tokofia.

Ko tou olo ki moana o nonofo mai ai $i$ totatou 'ü fo' $i$ vaka

kae tuku mai ia te kau tama kāuga

ki te matafenua pea toe liliu ifo.

'Iloga 'aē 'e mu'a ifo kae muli 'au tagata pea koutou mamate fuli."

'Ui age pē ia e Maka fai ia ki ai.

Pea hōhō mai leva Toke: "Maka ko au ia 'aē, ko toku 'aè temi.

Tuku kau'alu.

Kä lau mai pē ia 'aē ko te kamata pea ko au 'aē ka kāuga.

Fakahā kiā Tu’i Pulotu ko tona tokotolu pe au mu'a tokotahi."

Hake pē 'aia pe 'ui mai e Tu'i Pulotu : fai ki ai.

Ko te tahi, lua, tolu pē 'aia, hagē te lalaga ake a te Toke 'alu ia 'aia o tau ki te matafenua pea toe liliu ifo pea toe felāve'i mo te kau tama kāuga o 'alu ifo ki vaka.

Tali e Tu'i Pulotu oiane tā ko tatatou 'ū kau kāuga nei ko te me'a noa 'osi.

Nonofo ai nonofo ai pea ha'u te kau kāuga

Pea lea mai Tu’i Pulotu Maka, kua ke mālō pea kua ke ma'uli.

Kei toe te tahi faiva ko te uku.

Vaka'i mai hau tahi fa'a uku mo taku tokotolu 'aenī'e olo o nonofo i lalo.

Nonofo ai kae tou talitali tatou i 'oluga nei o lama :

pe ko ia 'aè 'uluaki mafuta ake kua mate.

Tali 'aia e Maka 'e fai ia ki ai Tu'i Pulotu, mais laisse-moi aller voir par derrière du côté des préparatifs du festin, laisse-moi faire."

"Entendu!"

Toke alla prendre un petit cochon pour leur repas

tandis que Mapula allait se placer à l'arrière des vivres du festin.

Mapula se mit alors à dévorer tous les vivres, les cochons,

les tubercules, les légumes cuits et même les feuilles de bananier cuites.

Tout fut englouti.

Puis, il alla se baigner à la mer afin de bien digérer.

Les gens du pays étaient stupéfaits.

Tu'i Pulotu ne savait plus quoi faire.

Après avoir digéré son repas, Mapula rejoignit l'assemblée.

Tu'i Pulotu les félicita :

"Maka, bravo pour ton courage, tu as la vie sauve !

Mais une autre épreuve t'attend : je vais te montrer ;

il s'agit de notre course de natation,

cette épreuve, c'est pour savoir qui est le plus rapide.

Voici mes trois nageurs, tu peux choisir le nombre de nageurs que tu veux.

Nous nous rendrons en mer à bord de nos pirogues

et nous laisserons les nageurs

faire un aller et retour complet entre les pirogues et la côte.

$\mathrm{Si}$ mon équipe arrive la première et tes hommes en dernier, vous devrez tous mourir."

Maka acquiesca.

Toke s'approcha de lui : "Maka, mon heure est venue,

Laisse, je vais y aller.

Lorsqu'on annoncera le départ, je partirai immédiatement à la nage.

Tu'i Pulotu doit être informé que je me présenterai seul contre ses trois nageurs."

Tu'i Pulotu donna son accord.

Un, deux, trois, Toke se précipita,

atteignit la côte, fit demi-tour,

croisa les nageurs du camp adverse et revint jusqu'aux bateaux.

Tu'i Pulotu s'exclama : “Oh la la, nos nageurs sont vraiment des bons à rien !"

Longtemps après en effet, ses nageurs arrivèrent.

Tu'i Pulotu ajouta : "Maka, tu as gagné et tu as donc la vie sauve.

Il reste cependant encore une autre épreuve : la plongée en apnée.

Choisis quelqu'un qui sache plonger pour concourir avec mes trois hommes à rester le plus longtemps possible au fond de l'eau.

Ils resteront au fond, et nous attendrons à la surface pour les surveiller :

celui qui remontera le premier sera un homme mort." Maka répondit : "Entendu, Tu'i Pulotu, 
he ko tou fenua 'e au fakalogo pē au ki he me'a 'e ke 'ui mai.

Tahi, lua, tolu pea 'ui age e Feke

ko au ia 'aenī ko to'oku temi.

'Alu te kau tama ki lalo, te 'alu ifo 'aia a nātou mo te 'alu ifo a te Feke.

Te 'alu ifo 'aia a te Feke o pā lafalafa i lalo

kae natou nonofo ai leva i lalo. Nonofo, nonofo.

Sio atu te Feke ia kua mapuhi te tahi tagata kua 'alu te pula ia.

'Alu foki lā te pula 'aia o 'asi i 'oluga.

Tali e Tu’i Pulotu: "kua fafā nei tau tamá Maka."

Tali e Maka : "e au mahalo."

Nofonofo ia nātou, 'asi ake ko te tagata a Tu'i Pulotu.

Kua faikehe 'osi fofoga ia o Tu'i Pulotu.

Toe nonofo. Sio mai ia te Feke 'aia kua mapuhi ake te tahi tagata.

Pea mapula ake 'aia.

Tali e Maka mahino nei ko tau tama 'aenī. Au mahalo !

\section{'Asi ake ko te tahi tagata a Tu'i Pulotu.}

Sio mai te Feke 'aia kua toe māmāpuhi ake te tahi tagata

pea alamai tona kave o puke.

O puke te tagata 'aenā o nā nonofo ai.

Fualoa te mate a te tagata kae nonofo pé ia mo ia,

pea hoki ina tuku ake.

'Alu ake lā te tagata 'aia ko te 'ū tagata fuli ia a Tu'i Pulotu.

Pea 'ui age e Tu'i Pulotu: "Maka, kua au lavā. Kua au tō. Kua ke ma'uli.

Tou hake." Hake ake mo te fu'u vaka ki 'uta.

Pea fai ai leva te tautālaga mo te fakamālō 'aē a Tu'i Pulotu, kiā Maka mo tana kau folau.

Pea 'ui age leva e Tu'i Pulotu : "ha'u lā Maka, ko koloa fuli o toku fenua

'e 'alu mo koe, mai te magisi ki te manu, mo me'a 'e ke fia fai ai, 'alu mo koe!

Kae ko'en̄̄ pē te fakamuli otāua felāve'i fai mu'a hata kava kava tau'ine.",

Tali e Maka : "fai ia ki ai."

Fai ai leva te kava ko te fo'i tāno'a foki.

Fo'i tāno'a 'aia 'e veliveli foki te fale ia 'aeni.

Fai 'aia te kava o fakahuhu'a o fonu 'osi.

Pea lea mai leva Tu'i Pulotu: "Maka, ’e ke fai lā hau palalau faka'osi ki si'au kau folau.

'Eau mahalo ko tatā fetaulaki pē 'aenī pea ke 'osi ai mo tou ma'uli.

Mole ke toe liliu.

Ko ia 'ae fai hau faka'amu ki tau kau folau pe kofea he 'aho

'e koutou sio ai ki tokotou fenua."

Tali e Maka kua lelei 'e au manatu au Tu'i Pulotu c'est ici ton pays, j'obéirai à tout ce que tu me demandes."

Un, deux, trois, Feke dit alors :

"Cette fois, c'est mon tour !"

Les jeunes gens descendirent au fond de l'eau, et Feke fit de même.

Il s'étala complètement au fond,

et les autres s'installèrent de même tout au fond. Longtemps, longtemps.

Enfin, le poulpe vit que l'un des jeunes commençait à perdre le souffle.

Des bulles d'air firent leur apparition à la surface.

Tu'i Pulotu dit alors : "Maka, ton co-équipier est sans doute fatigué à présent."

Maka lui répondit : “C'est possible."

Mais peu après, ils virent qu'il s'agissait d'un homme du Tu'i Pulotu.

Le visage du Tu'i Pulotu changea de couleur.

Ils attendirent encore. Le poulpe vit qu'un autre homme venait à perdre son souffle.

Des bulles d'air remontèrent à la surface.

Maka dit : "ce doit être l'un des tiens." - "Je le pense aussi."

Et c'était en effet un homme du Tu'i Pulotu.

Feke s'aperçut à nouveau qu'un autre commençait à perdre son souffle,

alors il le saisit de sa tentacule.

Il le coinça ainsi et ils restèrent tous deux au fond.

L'homme était déjà mort depuis longtemps, qu'ils restaient encore ensemble au fond,

et Feke finit par le relâcher.

L'homme remonta à la surface, tout comme l'avaient fait les autres hommes de Tu'i Pulotu.

Alors ce dernier dit: "Maka, j'ai perdu, je reconnais ma défaite. Tu as la vie sauve.

Repartons." Les pirogues retournèrent à terre.

Tu'i Pulotu fit beaucoup de compliments de félicitations à Maka et à ses compagnons de voyage.

Puis il lui dit: "Tiens, Maka, toutes les richesses de mon pays,

tu vas les emporter, qu'il s'agisse des tubercules, des bêtes, tout ce que tu veux, pars avec !

Mais pour mettre fin à notre rencontre, je voudrais que nous fassions un kava d'adieu."

Maka lui répondit : "Entendu !"

On prépara le kava dans un plat à kava.

Ce plat était plus grand qu'une maison comme celle où nous sommes.

Il fut rempli à ras bords.

Tu'i Pulotu prit la parole : "Maka, dis un dernier mot à tes compagnons.

Je crains que ce soit notre dernière rencontre avant que tu ne meurs.

Tu ne retourneras pas chez toi.

Vous pouvez seulement faire un vœu, toi et tes compagnons, pour qu'il existe un jour

où vous reverrez votre pays."

Maka répondit : "Je pense plutôt, Tu'i Pulotu, 
ko koe ia 'aē ke 'uluaki tau'inē mai kiā au.

Fai mai koe hau tau'inē.

Ko tatā māvete he au 'alu mole au nofo.

Kae ko tatā tau'inē faka'osi. Tā māvete 'aè ko te māvete faka'osi.

Pea lea mai Tu’i Pulotu fai ki ai, Maka.

Ko te kava a 'au mo tau kau folau.

Ke 'ago!

Kā mole 'ago pea ko te faka'osi leva 'aē o koutou i henī.

Lea mai Mapula : “Maka, takitahi pē koutou te ki’i ipu o inu."

'Osi inu pē' 'aia ia nātou pea hōhō atu leva ia Mapula ki te tāno'a.

Kamata fakalave mai tona laugutu i te tāno'a.

Tana 'au'au mai 'aia te huhu'a o te kava o 'alu fuli ki tona loto fatu.

Ha'u ai mo te fo'i maka tuki kava mo te 'ü ipu,

'Alu ai mo te tāno'a, ki loto.

Mole kei he tonu foki ia a Tu'i Pulotu.

'Ui mai e Tu'i Pulotu : "Maka, mālō, ha'u là o 'alu mo te 'ū koloa 'aē a Pulotu nei

he ko taku pūlou 'aē o talu ai.

Mole toe iai he pulotu.

'Alu lā o 'ave te mōlaga ki tou 'aliki.',

Pea ha'u ai te folau.

Pea 'osi 'aia leva te talanoa ki te folau 'aia ne'e 'alu ki Pulotu. que c'est toi qui va me dire au revoir le premier.

Tu vas me dire adieu.

Nous allons nous quitter car je vais partir, je ne resterai pas ici.

Ce sera un adieu définitif. Nous nous quitterons pour de bon."

Tu'i Pulotu lui répondit : "Comme tu voudras, Maka. Ce kava a été préparé pour toi et pour tes compagnons.

Bois-le jusqu'à la dernière goutte !

Si vous n'arrivez pas à le boire en totalité, ce sera votre fin à vous tous."

Mapula intervint alors: "Maka, ne prenez qu'une coupe chacun."

Après qu'ils eurent bu leur coupe, Mapula s'approcha du bord du plat à kava.

Il mit ses lèvres au bord du plat

et il en vida tout le contenu.

Il avala même la pierre pour piler le kava, ainsi que les coupes

et même le plat!

Tu'i Pulotu ne savait plus quoi faire.

Alors il dit: "Bravo, Maka, va-t-en avec toutes les richesses de Pulotu

car moi, je vais m'enterrer pour toujours.

Et Pulotu n'existera plus.

Va apporter tous ses présents à ton chef."

Les voyageurs retournèrent dans leur pays.

Et c'est ainsi que s'achève ce récit à propos d'un voyage à Pulotu.

\section{Visite au Pulotu}

Ko te talanoa 'aenī ki Pulotu ko te tagata ko Tōmasi i Futuna

pea ne'e fai tana gāue'aga i te kogame'a ko 'Asoa.

O fai ai tana gāue'aga pea ko tana gāue'aga 'e kaiha'a pē $i$ te pō fuli.

Kaiha'a te taló, kaiha'a te hopá, kaiha'a te 'ufí.

'E' 'alu ake ai pē te tagata ne'e kaiha'a ai pē tana gāue,

mole hala he pō mo te kaiha'a.

Pea 'alu leva te tagatá o moe ai i 'Asoa.

'Uluaki pō pē o'ona, pea fokifā te tagata kua 'alu age kiā ia.

O nà palalau. Pea 'ui age leva e te tagata

“Tōmasi 'e ke ha'u koa lā, 'e iai he me'a 'e 'iloga i tau gāue'aga?’'

“'Ei, ko taku gāue'aga 'e to'ona pē ia i te pō fuli.

Ko toku fusí mo taku gāue'aga 'ufí peamo taku taló,

'e au ha'u au pea 'e kaiha'a pēia.

Pea he' eki au ma'u au pē ko ai.",

Pea 'ui age leva e te tagata ko au, ko au'aia.

Pea ke ha'u mu'a koe ke tā olo ke ke sio, ke ke sio ki Pulotu.
Voici l'histoire de Pulotu : il était une fois à Futuna un homme, Tomasi,

qui avait un champ du côté d'Asoa.

Il s'occupait de ses plantations et, chaque nuit, quelqu'un venait le voler.

On lui volait des taros, des bananes, des ignames.

À chaque fois qu'il allait dans ses plantations, il constatait un vol,

Il n'y avait pas une nuit sans vol.

L'homme décida d'aller dormir à Asoa.

Dès la première nuit, un homme surgit devant lui.

Ils discutèrent. L'homme lui demanda :

"Tomasi, quelle raison te pousse donc à venir dans ta plantation?"

'Eh, c'est parce que c'est mon propre champ et qu'il est pillé tous les soirs,

qu'il s'agisse de mes bananiers, de mes ignames ou de mes taros,

je constate à chaque fois des vols.

Et je n'ai pas encore trouvé le responsable."

L'homme lui dit : "c'est moi, c'est moi-même !

Mais viens donc avec moi visiter Pulotu." 
Pea taki ifo pē ia e te tagata. O nā olo ifo.

Pea nā olo ifo 'aia anāua o a'u ki ava pea nā fakalava i te matafenua ('aē ha'u ki te mala'e vakalele).

Nā omaomai pē nāua pea fokifā pē ia 'e 'ui age e te tagata

“Tā olo hē !’ Pea sio atu pē ia ki te pū i te matame'a.

Ulu ai te tagata pea ulu atu mo ia.

Olo atu nāua 'e haha'i te kogame'a 'aia.

Pea ko te kau Futuna pé foki ia. Nofo ai.

Kae ko te 'ù magisi pèe'e tou kai i Alo, ko ia pēe 'e natou kai ai

te taló, peamo te niú, te magisi fuli.

Pea kai mo te moá mo te iká.

Nonofo ai ia nātou. 'Aho tolu.

Pea fêia age leva e te tagata: "Tōmasi ko koe foki 'e le'o i te 'aho nei.

'E le'o i totatou kogame'a." "Kae olo koutou ki fea?',

“'E olo mātou o gelu. Pea hoki matou omai. 'E au fakalogo pē au

kapau 'e ko au ia 'aē 'e le'o, pe au fakalogo foki pē au.' “'ēi, le'o koe. Pea ko te me'a pēe e fakahā atu kiā koe, 'e pülo'i koe e te kumete.

'E fakapūlo'i ia koe e te kumete. Pea 'ana na'a ke hue'i.

Hoki matou ha'u pē pea hue'i."'

'Ui age e Tōmasi fai ia ki ai.

Pūlo'i leva ia kae mole leva ina 'ilo'i foki ia pe ne'e toe olo te haha'i ia i fea 'aē foki o te kogame'a.

Ta'ana ia pūpūlou kua fia sio ia ki tu'a.

Pea ina lagalaga'i leva ia te tapa o te kumete.

O lagalaga ia sio ifo pè ia ki te haha'i o Alo.

Sio ifo ia 'e ha'ele ake pē nātou mo te feolo'aki 'aē mo anatou 'àmoga i Alo.

Pea pūlou. Logo ake pē ia kua logoa'a te kogame'a.

Ko tā ko te tau ake 'aia o te gelu.

Olo ake 'aia nātou o kai me'akai.

'Osi te kai me'akai pea 'ui age leva e te tagata

Tōmasi, hoki tā olo leva kau 'alu o tuku koe, ke ke 'alu koe ki Alo.

'Ui age e Tōmasi :" io, fakalogo pē ia kiā koe!'

'Aia là o natou momoe.

Tu'u ake ai te tagata o fafagu Tōmasi ke nā olo.

Nà olo pē nāua 'aia o a'u ki te matafenua.

Hū atu ia nāua ki tu'a. Pea fekau leva ia e te tagata "'alu lā koe Tōmasi !"

Kae mamaha te tai, ko te tai uhu,

kae sio ake ia ki te pū 'aè ne'e nā ulu mai ai 'e mole kei he pūia.
L'homme entraîna Tomasi avec lui et ils partirent. Ils se mirent en route et parvinrent à la passe, traversèrent le chemin du bord de mer (celui qui mène à l'aéroport).

Ils continuèrent leur route quand tout à coup, l'homme dit :

"Entrons là." Et Tomasi vit un trou sur le bord du rivage

dans lequel l'homme s'engouffra, alors il y entra aussi. Ils arrivèrent à un endroit rempli de monde.

Et il n'y avait que des Futuniens. Ils s'installèrent là.

La nourriture était la même qu'à Alo :

taros, coco, tubercules en tout genre.

Et il y avait aussi du poulet et du poisson.

Ils restèrent là trois jours.

Puis l'homme dit ainsi : "Tomasi, aujourd'hui, ce sera ton tour de garder la maison.

Tu vas garder notre endroit." "Mais où allez-vous donc ?", demanda Tomasi.

"Nous allons à la pêche, puis on sera de retour." "Entendu,

et si c'est mon tour de garde, j’obéirai."

"Oui, c'est ton tour de garde. Et il faut que tu saches que tu dois être recouvert d'un kumete $^{3}$.

Ce plat va te recouvrir. Et tu ne devras l'enlever en aucun cas.

Nous l'ôterons dès que nous serons de retour."

Tomasi déclara : "Qu'il en soit ainsi !"

Il se recouvrit du plat, sans savoir seulement où pouvaient bien aller les gens de cet endroit.

Après être resté sous le kumete pendant quelque temps, il eut envie de regarder à l'extérieur.

Alors il souleva doucement le bord du kumete.

Et quelle ne fut pas sa surprise d'apercevoir les gens d'Alo.

Il les voyait marcher ici et là avec leurs charges de nourriture!

Il laissa retomber le plat. Il entendait du bruit autour de lui :

Eh ! C'était le retour des pêcheurs.

Ils arrivèrent et se mirent à table.

Après le repas, l'homme vint lui dire :

"Tomasi, nous allons partir tous les deux, je vais te ramener à Alo."

Tomasi répondit : "Entendu, c'est comme tu le veux !" Voilà, ils allèrent d'abord dormir un peu.

Puis l'homme se leva, réveilla Tomasi pour partir.

Ils se mirent en route pour le bord de mer.

Ils émergèrent, et l'homme salua Tomasi :

"Au revoir !"

La marée était basse, c'était la marée montante du matin,

il vit que le trou par lequel ils étaient sortis avait disparu.

3. Récipient allongé en bois dans lequel on prépare l'amidon, le curcuma, le lait de coco, etc., et qui sert aussi de petite embarcation 
Pea ha'u leva ia i te mamaha foki 'aē o te tai.

Ha'u lā ia kae ko te nofo ia o tonātou 'api i Alo kua mate.

Kua tonu lā ia ko te 'aho 'aia 'e fai ai tona ia pogipogi, 'aè o Tōmasi.

Kua 'afu lā te 'atu' 'umu ia.

Ha'u ha'u 'aia peamo te vavalo age foki 'aē o te tagata 'e ha'u i te matafenua

Pea kua 'ui fuli foki ia e te haha'i 'e ko'e hagē ko Tōmasi 'aè 'e ha'u?

Ha'uha'u'aia pea fêia age leva e te 'aliki 'aē ko Sāgogo. 'Ui age leva e te 'aliki tou nonofo là o fakalogo ki tana ha'u.

Ka ha'u 'ànai 'aēo 'alu ake o ulu mai i te tau'á pea kei ma'uli, ko Tōmasi.

Pea ka ha'u leva ia o ulu mai i te mu'a fale, 'aè 'e tou nofo ai tātou,

pea tā kua mate.

Ha'u pē 'aia ia te tagata, ha'u pē ia o 'alu ake o kumi mai te tau'á,

ha'u o nofo mai ai.

Pea fai atu te fakamālō.

Mālō Tōmasi, pea io mai foki ia. 'Aēo nātou palapalalau ai.

Pea hoki 'ui age leva e Sāgogo Tōmasi, 'e ke ha'u lā ko te 'umu 'aenā 'e fai i ma'uga ko tou ia pogipogi.

Tamātou nofo, kua ke mate koe!

Pea hoki fakamatala e Tōmasi he'eki au mate!

Ne'e 'ave au ia ki Pulotu. 'Ave au ia e te tagata ki Pulotu.

Ha'u 'aia o ina fakamatala age foki, tona 'ave peamo tona 'aumai 'aē o tuku.

Ko tona pè tuku mai ki tu'a pea puli te pū 'aē ne'e nā feulufi ia mo te tagatá.

Pea 'osi ai foki te talanoa ia.

\section{BIBLIOGRAPHIE}

Bataillon Pierre, 1932. Langue d'Uvea (Wallis). Grammaire-dictionnaire uvea-français. Dictionnaire français-uvea-anglais, Paris, Librairie orientaliste Paul Geuthner.

Burrows Edwin G., 1936, Ethnology of Futuna, Honolulu, Bulletin of the Bernice P. Bishop Museum 138 ,

—, 1937. Ethnology of Uvea, Honolulu, Bulletin of the Bernice P. Bishop Museum 145.

FrimigaCCI Daniel et Maurice HaRdy, 1996. Rapport d'activité, Service de la Sauvegarde du Patrimoine, Wallis-et-Futuna, Programme de restauration et fouilles de sauvegarde.

Frimigacci Daniel, Muni Keletaona, Claire MoyseFAURIE et Bernard Vienne, 1995, La tortue au dos
Il revint sur la côte, facilité en cela par la marée basse. Il arriva chez lui à Alo et vit que dans sa maisonnée, il $\mathrm{y}$ avait eu un mort.

Et c'était justement le jour du repas de deuil, le repas de deuil pour Tomasi!

Les fours, alignés, étaient prêts.

En arrivant sur les lieux, tout le monde le dévisagea.

Les gens présents se dirent: "Mais n'est-ce pas Tomasi qui arrive?"'

Tandis qu'il approchait, le chef Sagogo dit ainsi, il dit: "Attendons voir ce qu'il va faire.

S'il vient et s'asseoit du côté des préparateurs de kava, c'est qu'il est encore en vie, c'est que c'est véritalement Tomasi.

Mais s'il vient se placer devant du côté des chefs

alors eh bien ! c'est qu'il est bien mort !'

L'homme alla se placer du côté des préparateurs du kava

et s'installa.

On échangea les salutations.

"Bonjour, Tomasi", et Tomasi répondit de même. Et tous se mirent à bavarder.

Sagogo lui dit : "Tomasi, te voilà,

alors que s'organise à la montagne un repas de deuil en ton honneur.

On a cru que tu étais mort !"

Tomasi leur expliqua : "Je ne suis pas encore mort !

On m'a emmené au Pulotu. Un homme m'a emmené au Pulotu.'

Il raconta alors comment on l'avait emmené, puis ramené et laissé.

Il raconta que dès qu'il avait atteint la surface de la mer, le trou avait disparu, celui par lequel lui et l'homme de Pulotu s'étaient engouffrés.

C'est ainsi que s'achève cette histoire.

moussu. Ko le fonu tu'a limulimua. Textes de tradition orale de Futuna, Peeters-Selaf, Langues et cultures du Pacifique 11.

Geraghty Paul, 1993, Pulotu, Polynesian Homeland, Journal of the Polynesian Society 102, 4, Pp. 343384.

Henquel Joseph, 1973 [1910], Talanoa $i$ 'Uvea nei, manuscrit rédigé entre 1896 et 1910, publié par la Direction de l'enseignement catholique de Wallis.

Hovdhaugen Even, 1987, From the land of Näfanua, Norwegian University Press, The Institute for Comparative Research in Human Culture.

MAYER Raymond, 1976, Les transformations de la tradition narrative à l'île Wallis (Uvea), Paris, Société des Océanistes, Publications de la Société des Océanistes 38, $311 \mathrm{p}$, 
Moyse-FAurie Claire, 1995, Le xârâcùù, langue de Thio-Canala (Nouvelle-Calédonie). Éléments de syntaxe, Peeters-Selaf, LCP 10 (avec l'histoire de Deekwâxiti),
Servant Louis Catherin, 1995, Écrits, Paris, Éditions Tequi. 